\title{
Candidate main-field models for the Definitive Geomagnetic Reference Field 1995.0 and 2000.0
}

\author{
A. Chambodut ${ }^{1 *}$, B. Langlais ${ }^{2 \dagger}$, and M. Mandea ${ }^{1 \ddagger}$ \\ ${ }^{1}$ Laboratoire de Géomagnétisme et Paléomagnétisme, Institut de Physique du Globe de Paris, France \\ ${ }^{2}$ Laboratoire de Planétologie et Géodynamique, CNRS UMR 6112, Université de Nantes, France
}

(Received January 19, 2005; Revised July 14, 2005; Accepted July 14, 2005)

\begin{abstract}
Two geomagnetic main-field models for epochs 1995.0 and 2000.0 were proposed as candidate models for DGRF 1995 and DGRF 2000. A main-field model was derived for epoch 2000.0, using the high-quality data provided by the Ørsted satellite around this epoch. Since no high-quality satellite vector measurements of the magnetic field were acquired between 1980 and 1999, our approach was to extrapolate this 2000.0 accurate model back to 1995. To do this we produced a secular-variation model for the time-span 1995-2000, from ground measurements. The models obtained were incorporated into DGRF 1995 and DGRF 2000 as part of the 9th generation of the IGRF in 2003.
\end{abstract}

Key words: Main field, satellite and observatory data, DGRF 1995 and 2000.

\section{Introduction}

The launch of the Ørsted satellite has considerably changed the way magnetic models are constructed, or more specifically, the way magnetic measurements are selected. For the 8th generation of IGRF (previously IGRF 2000), main-field candidate models relied on spatially incomplete datasets, resulting in errors that were too large to be ignored. The situation is now different, as an unprecedented and geographically complete dataset is available. Due to the very large number of magnetic measurements, it is possible to apply very strict data selection criteria. In this context, we chose to derive a model for epoch 2000.0 from selected measurements provided by the Ørsted satellite at the time of this study. Then, this model was extrapolated back to epoch 1995.0 using a secular-variation model derived from monthly mean observatory data.

\section{A Candidate Main-Field Model for the DGRF 2000 and a Secular-Variation Model for 2000- 2005}

Our candidate main-field model for DGRF 2000 was computed using selected vector and scalar one-second measurements of Ørsted satellite mission, over the 1999-2002 period. The data were downloaded from the Ørsted Science Data Center (see the web page: http://web.dmi.dk/fsweb/ projects/oersted/SDC/oersted.html).

*now at: Department of Applied and Industrial Mathematics, Universität Potsdam, Germany.

${ }^{\dagger}$ Formerly : NAS/NRC at NASA/GSFC, code 921, Greenbelt, MD, USA.

${ }^{\ddagger}$ now at: GFZ Potsdam, Germany.

Copyright (c) The Society of Geomagnetism and Earth, Planetary and Space Sciences (SGEPSS); The Seismological Society of Japan; The Volcanological Society of Japan; The Geodetic Society of Japan; The Japanese Society for Planetary Sciences; TERRAPUB.

\subsection{Data selection criteria}

In order to avoid outliers, a first check was done on the raw dataset, using a procedure based on the first and second time derivatives (Langlais et al., 1999). A three-step standard selection was done in order to minimise the external magnetic field contributions. First, the effects of field-aligned currents were reduced, by using only scalar measurements above $50^{\circ}$ absolute geomagnetic latitude. Then, a selection was done with respect to magnetic activity indices. Only data corresponding to periods characterised by the following geomagnetic indices were kept: $K p(t) \leq 1+$ and $K p(t-3 \mathrm{~h}) \leq 2-;|D s t(t)| \leq 5 \mathrm{nT}$ and $|d D s t(t) / d t| \leq 3 \mathrm{nT} / \mathrm{h}$. Finally, a local-time selection was applied. Only measurements acquired in the "real shadow side" of the Earth (data acquired when the satellite is in the shadow of the Earth) were selected, which is a more restrictive criteria than the classical local time 22:00-06:00 selection (Chambodut et al., 2003).

The number of data was important (see Table 1). In order to ensure a data distribution in space and time that is as regular as possible, we decimated data over the globe for each two-month interval. An equiangular grid of $5^{\circ} \times 5^{\circ}$ was defined. One measurement per bin was then randomly selected. These two-month data files were merged. In this way, we reduced the initial number of data but maintained a regular distribution in space and time.

A homogenisation scheme was then used. An equiangular grid of $3^{\circ} \times 3^{\circ}$ was defined and up to ten measurements per bin were randomly selected. For absolute geomagnetic latitudes below $50^{\circ}$, scalar measurements were used only if vector measurements were missing. In order to counterbalance the denser data distribution near the poles, a geographic weighting scheme was applied, by weighting each measurement by the sine of its colatitude. We combined the geographic weighting scheme with an anisotropic one based on the attitude uncertainty. This is related to a poorer 


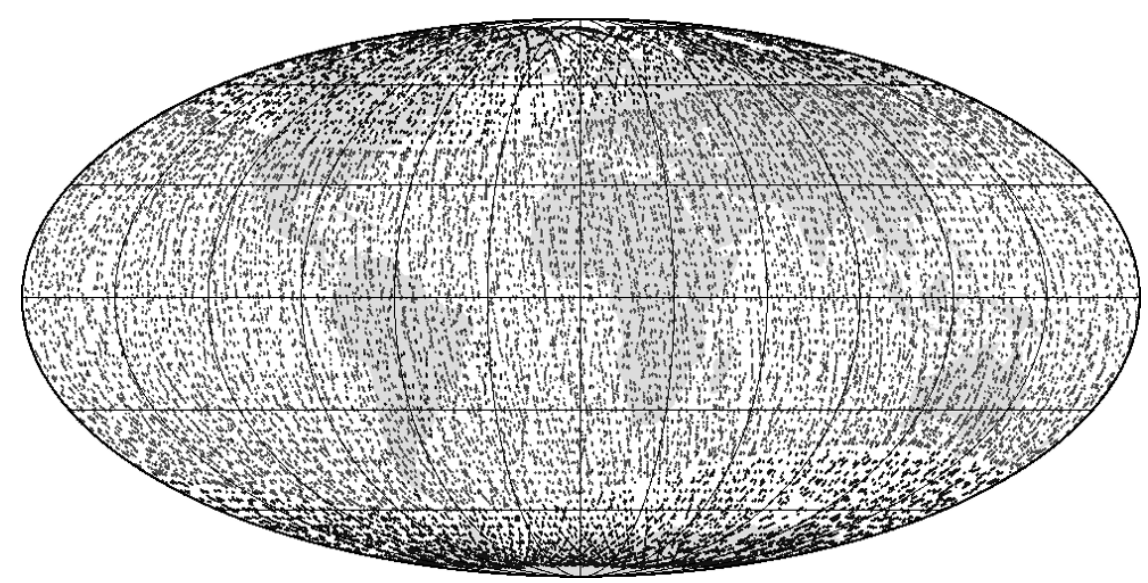

Fig. 1. Geographical distribution of Ørsted satellite data used in computing the final model (after homogenisation).

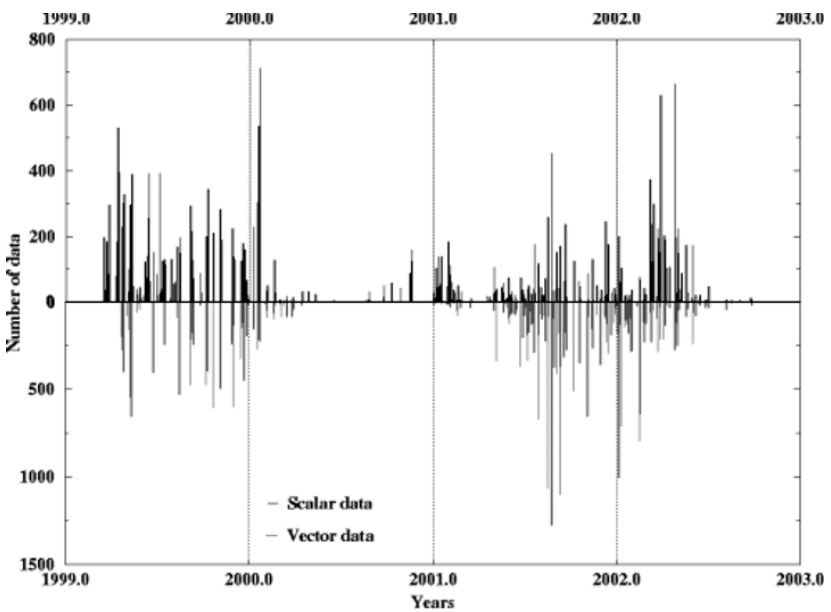

Fig. 2. Temporal distribution of scalar (top) and vector (bottom) Ørsted satellite data used in computing the final model.

Table 1. Evolution of the number of data through successive selections $\left({ }^{a}\right.$ preliminary model, ${ }^{b}$ final model $)$.

\begin{tabular}{lcc}
\hline & Vector triplets & Scalar \\
\hline $\begin{array}{l}\text { after main selection } \\
\text { (Dst, Kp, "real shadow side") }\end{array}$ & 1570107 & 1236184 \\
\hline $\begin{array}{l}\text { after reduction of the } \\
\text { number of data at random }\end{array}$ & 48301 & 38497 \\
\hline after homogenisation $^{a}$ & 39093 & 25723 \\
\hline $\begin{array}{l}\text { after test on residuals } \\
\text { (RMS } \leq 5 \text { nT) }\end{array}$ & 55090 & 52460 \\
\hline after homogenisation & & \\
\hline
\end{tabular}

determination of the rotation angle around the pointing axis of the Star Imager (SIM) for the Ørsted satellite (Holme, 2000). Error angles were set to 10 arcsec for the direction along the SIM axis, and to 60 arcsec for the perpendicular direction.

\subsection{Modelling}

The candidate main-field model for epoch 2000.0 was obtained in a two-step procedure. Using a selected and homogenised dataset, a first rough model was computed up to degree/order 29 for internal field, 10 for secular varia-
Table 2. The root mean square residuals of the final model.

\begin{tabular}{rll}
\hline X rms & 2.5 & $\mathrm{nT}$ \\
Y rms & 3.0 & $\mathrm{nT}$ \\
$\mathrm{Z} \mathrm{rms}$ & 2.7 & $\mathrm{nT}$ \\
\hline vector total rms & 2.6 & $\mathrm{nT}$ \\
\hline scalar total rms & 2.8 & $\mathrm{nT}$ \\
\hline total rms & 2.7 & $\mathrm{nT}$ \\
\hline
\end{tabular}

tion and 2 for external field, with a first degree/order $D s t$ dependence. The Gauss coefficients were estimated by an iterative least-square fit. They were then used to predict the magnetic field for all measurement locations. This allowed us to refine the data selection, by only keeping data for which absolute residuals were lower than $5 \mathrm{nT}$ for each component.

We applied the homogenisation procedure to the new selected dataset, introducing new data into bins which were previously depleted by the $5 \mathrm{nT}$ selection criteria. Geographical and time distributions of the resulting dataset are shown on Figs. 1 and 2, and the numbers of scalar and vector measurements, before and after the selection with respect to the preliminary model, are given in Table 1 .

The spherical harmonic analysis was done up to degree/order 20 for internal field, 10 for secular variation and 2 for external field, with a first degree/order Dst dependence. Secular-variation terms for $n=9$ and $n=10$ were imposed, using values of model Ø19992000_SVM (Langlais et al., 2003). The root mean square residuals of this final model are given in Table 2. Our candidate models for DGRF 2000 and for IGRF 2000-2005 secular variation were truncated versions of this final model, up to degree/order 13 for the main field and up to degree/order 8 for the secular variation.

\section{A Candidate Main-Field Model for the DGRF 1995}

Our candidate main-field model for DGRF 1995 was calculated retrospectively from candidate main-field model for epoch 2000, by applying a secular-variation correction over the 1995-2000 period. Details of the secular-variation 
- 112 Contributing Observatories

$\Delta 11$ Contributing Observatories with high residuals (at least 2 components over 2 nT/year)

$\checkmark 4$ Contributing Observatories with high residuals (at least 2 components over 3 nT/year)

$\oplus 15$ Rejected Observatories (incomplete data, jumps, spurious time series, residuals)

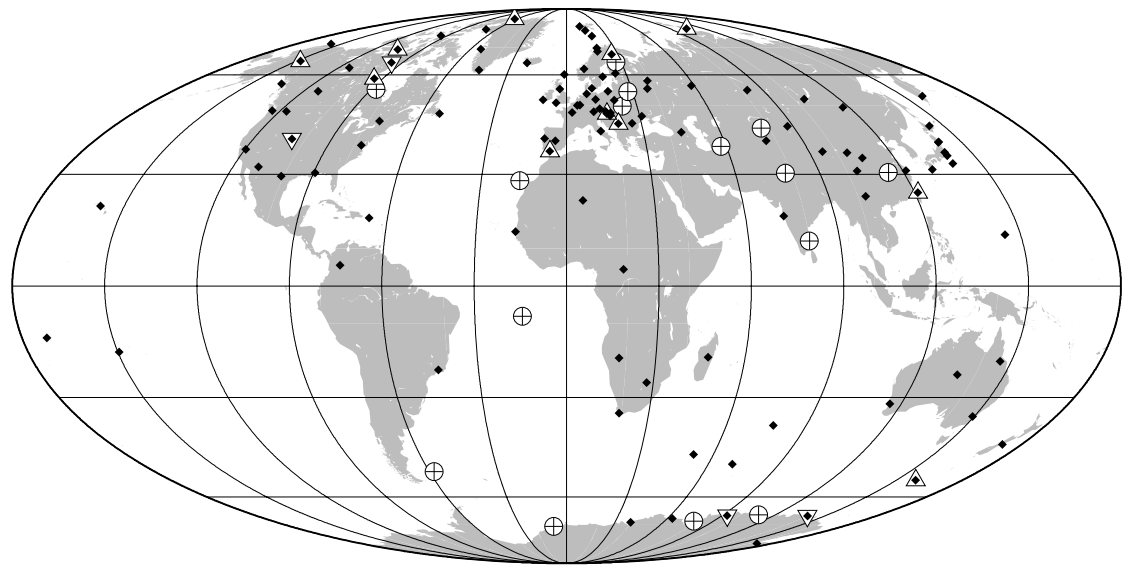

Fig. 3. The 112 observatories used in the secular-variation model.

model, based on observatory annual means, are now presented.

\subsection{Observatory data}

First, annual main-field models were computed, using observatory annual means. These models were thereafter used to derive annual secular-variation models. These main-field models were based on a constant geographical observatory distribution over the entire 1995-2000 interval. Time series from 1994.5 to 2000.5 were used, but time series back to 1979.5 were examined whenever possible, in order to check the time series measurements consistency (by plotting the time series of the annual means). This also helped us to double-check the crustal biases (see below). Only 112 observatories provided annual mean values from 1994.5 to 2000.5. A jump was applied for TUC in 1996, and data for SYO were interpolated for 1997.5 and 2000.5. The final observatory geographical distribution is plotted and described in Fig. 3.

\subsection{Modelling parameters}

3.2.1 Crustal corrections Satellite-based models computed by Langlais et al. (2003) were used to predict values for epoch 2000.0 at each observatory location. The models used were denoted Ø19991112 and Ø20000102. The crustal corrections were derived by comparing these predicted values, up to degree $n=13$, and the 1999.5 and 2000.5 mean values. No significant difference was found when comparing these biases with biases for 1980.0 based on the M19791112 and M19800102 models of Langlais et al. (2003).

3.2.2 Weights Because of the poor data distribution (Northern hemisphere lands better covered), it was necessary to use an adequate weighing scheme that counterbalances this uneven geographical distribution. The complete weighting scheme is described in Langlais and Mandea (2000): for each observatory, a mean index is computed, inversely proportional to the distance between this observatory and the closest one in each of the four North-East, North-West, South-West and South-East quarters. A maximum distance, equal to: $2 \pi a / 2 n$ (where $a=6371.2 \mathrm{~km}$,

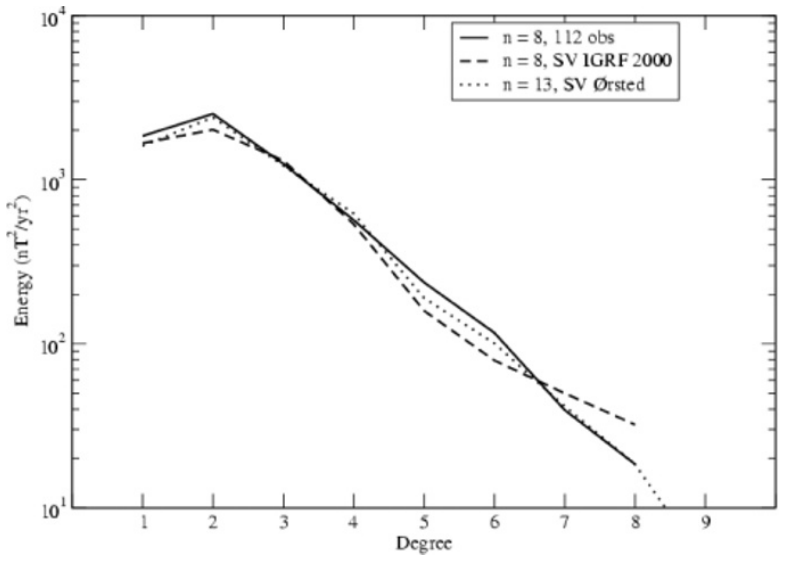

Fig. 4. Comparison between secular-variation models computed for this study or already published.

the mean radius of the Earth, and $n$ the maximum degree of the spherical harmonic expansion) was set so that isolated observatories were not assigned weights that were too high. Each annual main-field model was based on the same weighted data distribution.

\subsection{Modelling}

Seven main-field models were obtained, up to degree and order 8. Associated rms values between annual means and predictions were about 40/45 nT each year. Without crustal corrections, these rms values were about $230 \mathrm{nT}$. From these seven main-field models, six secular-variation models were computed (from 1995.0 to 2000.0). In order to test these models, a sum of the six annual secular-variation model for period 1994.5-2000.5 was derived, which was used to predict the observed variations. The rms differences between observations and predictions were equal to $1.63,1.71$ and $1.58 \mathrm{nT} / \mathrm{yr}$ for the $\dot{X}, \dot{Y}$ and $\dot{Z}$ components, respectively. In only four observatories (BLC, BOU, DRV and MIR) the differences between observed and predicted variations were larger than $3 \mathrm{nT} / \mathrm{yr}$ for two of the three components (see Fig. 3). 

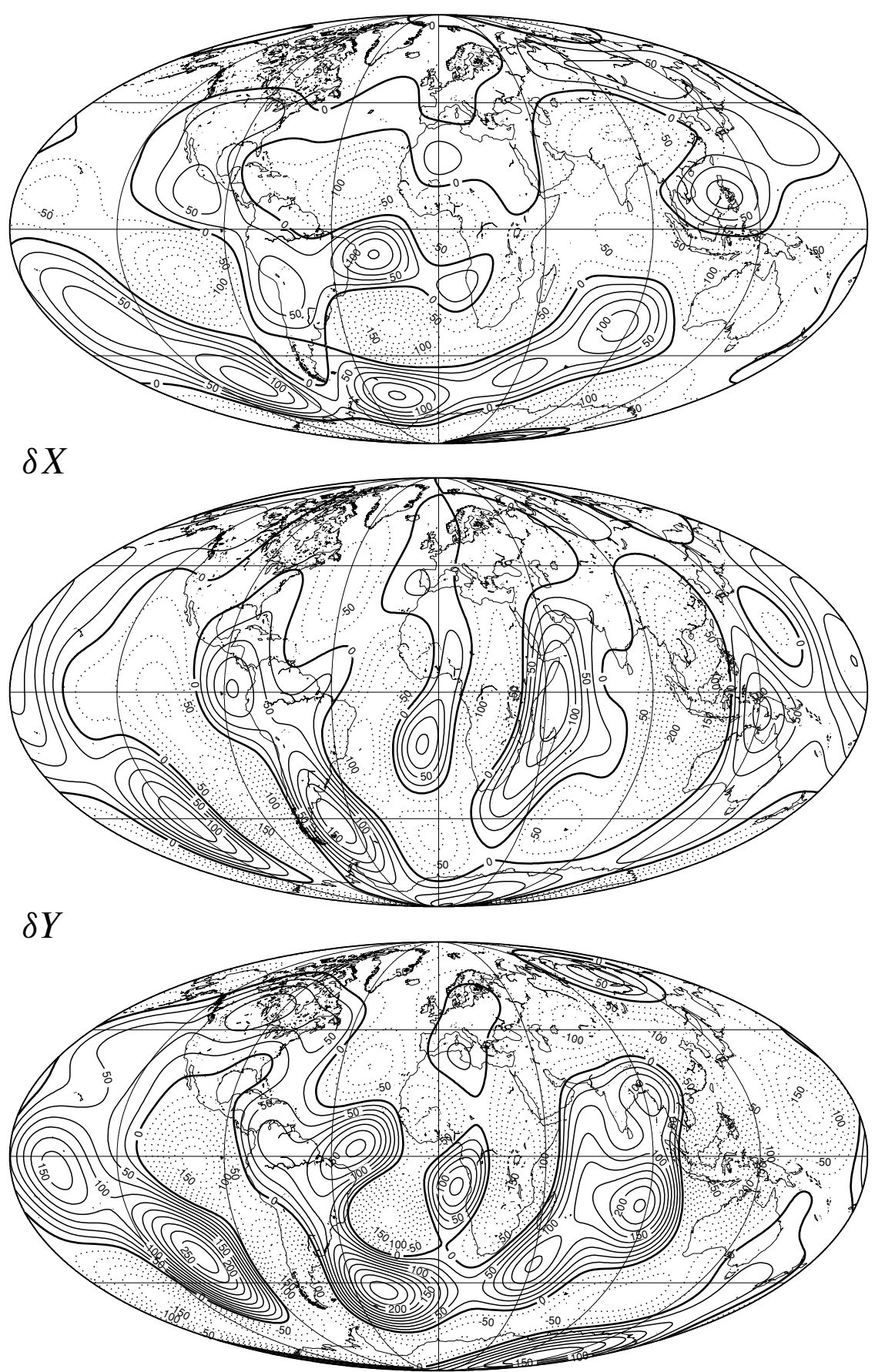

$\delta Z$

Fig. 5. Differences in nT between model IGRF 1995 and the proposed DGRF model for epoch 1995. Contour lines are every $\pm 25 \mathrm{nT}$ (dashed lines for negative values; continuous lines for positive values).

Finally, a mean 1995-2000 secular-variation model was computed up to degree and order 8 . For degrees 9 and 10 the a priori secular-variation coefficients were imposed. Secular-variation energy spectra of this final model is compared to some others, in Fig. 4. The overall pattern is similar, but some large differences appear particularly with the secular-variation model of the IGRF 2000. The model we proposed is obviously nearest to the model Ø19992000_SVM (Langlais et al., 2003) used in the present study as a priori secular-variation model. Applying this secular variation to our candidate main-field model for epoch 2000, we obtained the candidate main-field model for 1995.0.

\section{Discussion and Conclusion}

Two candidate main-field models for DGRF 1995 and 2000, as well as a candidate secular-variation model for epoch 2000-2005 are presented. Figures 5 and 6 present the differences between IGRF 1995 model and our proposed DGRF 1995 model, and IGRF 2000 model and our proposed DGRF 2000 model, respectively.

The large differences for the epoch 1995.0 are likely to 

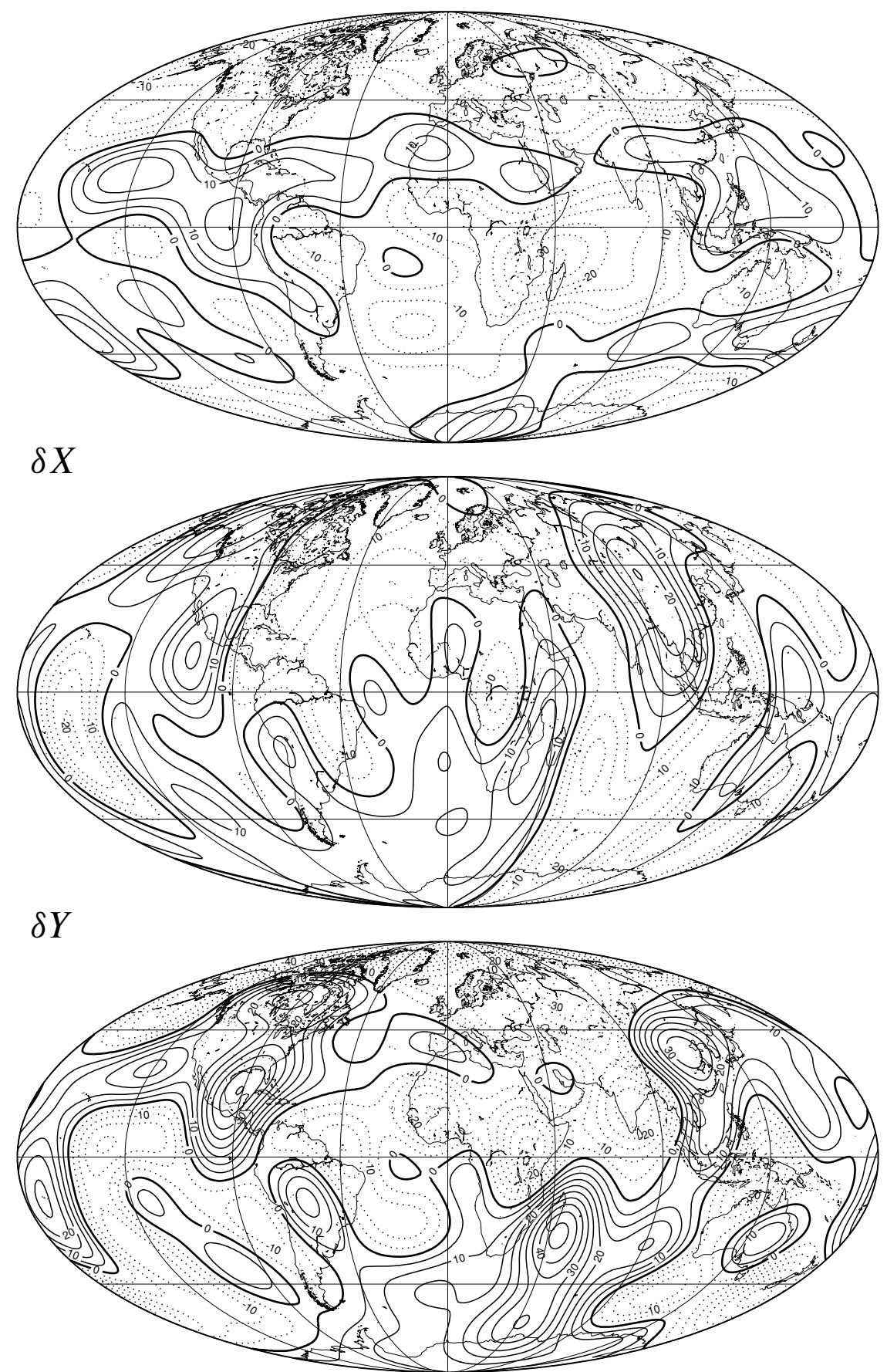

$\delta Z$

Fig. 6. Differences in nT between model IGRF 2000 and the proposed DGRF model for epoch 2000, truncated to degree 10 to be the same as IGRF 2000. Contour lines are every $\pm 5 \mathrm{nT}$ (dashed lines for negative values; continuous lines for positive values).

be due to the fact that mainly land measurements were used in deriving the corresponding IGRF. The plots (Fig. 5) show that the differences vary from about $\pm 300 \mathrm{nT}$ in $\mathrm{Z}$ component to $\pm 150 \mathrm{nT}$ in $\mathrm{X}$ or $\mathrm{Y}$ component. The spatial distribution of residuals reflects the observatory positions at the surface of the Earth. As an example, above Europe, residuals are negligible, while they dramatically increase in South Pacific or Atlantic area, where there are fewer or no observatories.

For epoch 2000.0, the residuals are lower. The plots (Fig. 6) show that the differences vary from about $\pm 60 \mathrm{nT}$ in $\mathrm{Z}$ component to $\pm 20 \mathrm{nT}$ in $\mathrm{X}$ or $\mathrm{Y}$ component. The differences between models for epoch 1995.0 are about one order of magnitude greater than the differences between models for epoch 2000.0. This is not surprising as accurate vector satellite measurements were used for IGRF 2000.

The satellite information has greatly improved our knowledge of the recent short-term secular variation. However, there is still the problem of extrapolation into the past five years, as the secular variation itself has a temporal variation of a few $\mathrm{nT} / \mathrm{yr}^{2}$. Our candidate models were incorporated into the 9th generation IGRF produced in 2003. 
Acknowledgments. We would like to thank our editor, Susan Macmillan, and Nils Olsen and Angelo De Santis for their constructive remarks and comments. We are pleased to thank the staff of the geomagnetic observatories and of Ørsted satellite mission for supplying high-quality data. All maps have been plotted using the General Mapping Tool Software (Wessel and Smith, 1991). Benoit Langlais was supported by a NAS/NRC postdoctoral fellowship. This is IPGP contribution number 2068.

\section{References}

Chambodut, A., J. Schwarte, B. Langlais, H. Luhr, and M. Mandea, The selection of data in field modeling, Proceedings of the 4th OIST meeting, 2003.

Holme, R., Modeling of attitude error in vector magnetic data: application to Ørsted data, Earth Planets Space, 52, 1187-1197, 2000.
Langlais, B. and M. Mandea, An IGRF candidate main geomagnetic field model for epoch 2000 and a secular variation model for 2000-2005, Earth Planets Space, 52, 1137-1148, 2000.

Langlais, B., P. Ultré-Guérard, C. Vernin, M. Mandea, Y. Cohen, and G. Hulot, Ørsted: IPGP commissioning of the OVH magnetometer, technical report CNES, OERS_RP_0000_0031_IPG, 1999.

Langlais, B., M. Mandea, and P. Ultré-Guérard, High-resolution magnetic field modeling: application to MAGSAT and Ørsted data, Phys. Earth Planet. Int., 135, 77-91, 2003.

Wessel, P. and W. H. F. Smith, Free software helps map and display data, Eos Trans. AGU, 72, 441-448, 1991.

A. Chambodut (e-mail: chambodu@math.uni-potsdam.de), B. Langlais (e-mail: Benoit.Langlais@chimie.univ-nantes.fr), and M. Mandea (e-mail: mioara@gfz-potsdam.de). 